\title{
A 44-Year-Old Man with Profound Behavioural Changes
}

\author{
R. Laforce Jr, G.A. Kerchner, G.D. Rabinovici, J.C. Fong, B.L. Miller, W.W. Seeley, L.T. Grinberg
}

Can J Neurol Sci. 2012; 39: 527-530

At the age of 44 years, this man became progressively apathetic, disinhibited, obsessional and ritualistic. He set his alarm clock every two hours at night so he could go to the bathroom. He was fired from his job as an account analyst because he often fell asleep at work, and was unable to meet deadlines. There was profound lack of concern for his poor performance. He entered a neighbour's home when he found the door open and wandered around the house until he found the owners in their bedroom. Once described as a quiet and reserved man, he started going to strip clubs up to two times a day and walked up to strangers in restaurants to tell stories or comment on their appearance. He seemed to no longer respond to social cues, not waiting his turn to talk and walking up to two blocks ahead of his 70-year-old mother. He lacked warmth and was emotionally blunted. His level of initiative decreased, and he had to be encouraged to bathe and change clothes. He often missed the toilet when he urinated and defecated on the floor of his bedroom. A diagnosis of depression was made, but he was unresponsive to antidepressants.

The obsessional and ritualistic nature of his behaviour was especially striking. He watched the same video segments repetitively and told the same phrases over and over again. He repetitively washed his hands and became extremely regimented about the timing of his medications. He was severely obsessed with food, eating as many as 100-200 cough drops every day. He had a similar obsession with carrots, which he ate in a smaller quantity. He insisted on eating 'Taco Bell' at least four times a week, each time ordering the same meal. Once, he got up in the middle of the night to drive his mother's car to 'Taco Bell' even though his driving license had been revoked. He gained 50 pounds over a year.

Developmental and social history revealed that he reached all milestones normally and did well in school, obtaining a Bachelor's degree in finance. Prior to his illness, he had always been an amicable and productive employee. He dated women only sporadically but maintained a very close group of friends who supported him throughout the progression of his symptoms. Past medical history included hypothyroidism, hypertension, benign prostatic hypertrophy (BPH), pre-diabetes, and repair of a patent ductus arteriosus at 11 months-of-age which his brother also suffered from. He was allergic to sulfa drugs. His medications included a mood stabiliser for behavioural control (valproic acid $250 \mathrm{mg}$ BID, started in 2008), a 5- $\alpha$ reductase inhibitor for $\mathrm{BPH}$, levothyroxine, and anti-hypertensives. $\mathrm{He}$ never smoked, abused alcohol, or used recreational drugs. He had two healthy siblings. There was no family history of dementia.
Neurological examination revealed an alert, oriented, and fluent individual with a profoundly flat affect and monotonous voice. Behaviour was stereotyped and ritualistic. He interrupted conversation to describe the same scenes repetitively. This was mechanical and rote: 'Then he said..., and then she said...'. He frequently asked for cough drops, which he consumed continuously throughout the exam, one-at-a-time. Cranial nerves were unremarkable. Muscle strength, bulk and tone were normal. There was no bradykinesia, dystonia, fasciculation, myoclonus, or alien limb phenomenon. Sensory testing was intact. Reflexes were $2+$ and symmetric. Toes were downgoing to plantar stimulation. Coordination and gait were normal.

On neuropsychological testing, he showed marked attentional and executive deficits. He was able to repeat seven digits forward but only three backward. His performance on the Trail Making-B was slowed and he made 4 errors (see Figure 1C). There was evidence of difficulty learning and recalling both verbal and nonverbal information presented without a structured and contextual-rich environment, but he consistently benefited from cueing. Repetitive stories intruded into his responses and he often described the last scene of the movie 'Dumb and Dumber', although he was unable to explain why that scene was particularly funny to him. His performance on standard executive tests (i.e. verbal and design fluency, abstract reasoning, problem solving, Luria's 3-step sequence) was grossly impaired, and responses were perseverative. There was no difficulty with naming, repetition, reading, articulation, or comprehension. He was able to perform simple calculations. There was no evidence of visuospatial dysfunction.

Over the following two years he became increasingly inert and expressionless. Speech output was rare, hypophonic and echolalic. He did not initiate or plan actions spontaneously, and when he did so following verbal commands, his actions were perseverative. He could walk but showed mild gait difficulties. Despite mild dysphagia, he stuffed his mouth with food. He died suddenly by choking at the age of 47 , three years after the onset of his symptoms. He met all criteria for probable behavioural variant of frontotemporal dementia (bvFTD). ${ }^{1}$

From the Memory and Aging Center \& Department of Neurology (RL, GDR, JCF, BLM, WWS, LTG), University of California San Francisco; Stanford Center for Memory Disorders (GAK), Department of Neurology and Neurological Sciences, Stanford University School of Medicine, Stanford, California, USA.

Received September 19, 2011. Final Revisions Submitted February 8, 2012. Correspondence to: Robert Laforce Jr., UCSF Memory and Aging Center, 350 Parnassus Avenue, Suite 905, San Francisco, California, 94143, USA.

Email: rlaforce@memory.ucsf.edu 


\section{Clinical investigation}

Basic laboratory tests including blood count, ions, urea/creatinine, glucose, B12/folate, thyroid stimulating hormone (TSH), $\mathrm{Ca} / \mathrm{Mg} / \mathrm{Ph}$, urinalysis, hepatic enzymes, valproic acid, albumin, Venereal Disease Research Laboratory test (VDRL), Angiotensin-Converting Enzyme (ACE), overnight ambulatory sleep study, lumbar puncture (with 14-3-3, Abeta and tau), electromyogram (EMG), and electroencephalogram (EEG) were unremarkable. Magnetic resonance imaging (MRI) of the brain obtained shortly after the beginning of his disease revealed right greater than left atrophy in the frontotemporal areas as well as the caudate nucleus (see Figure 1). Genetic testing of APOE, MAPT A152T, CLU, SORL1, PICALM, GRN sequencing, MAPT, and fused in sarcoma (FUS) revealed no mutation.

\section{Neuropathology}

The brain showed severe atrophy of the frontal cortices, more pronounced in the right hemisphere (weight: $1330 \mathrm{~g}$ ). There was also moderate atrophy of the corpus striatum, particularly the $\mathrm{R}>\mathrm{L}$ caudate nuclei. Microscopic examination showed severe microvacuolation and astrogliosis in dorsolateral, orbito-frontal and anterior cingulate cortices. These areas also displayed remarkable neuronal loss. The same changes were observed to a lesser extent in the insula and mesial temporal regions. No vascular changes were identified. Immunohistochemistry was used to identify neurodegenerative disease-related inclusions. Microscopic examination showed inclusions positive for ubiquitin but not for transactive response DNA-binding protein (TDP-43), tau, alpha-synuclein or $\beta$-amyloid. Immunostaining for FUS revealed a moderate number of FUS-immunoreactive neuronal cytoplasmic inclusions and dystrophic neurites in the same regions displaying neuronal loss and microvacuolation (see Figure 2). ${ }^{2}$

\section{Discussion}

Accurate recognition of the underlying pathology based on specific behavioural profiles is one of the key goals in dementia care. In the recent years, the frontotemporal lobar degenerations (FTLDs) have been shown to classify into three main groups based on the major protein deposited in the brain (FTLD-tau, FTLD-TDP and FTLD-FUS). In a patient with bvFTD, the presence of neurological features such as motor neuron disease/amyotrophic lateral sclerosis (MND/ALS) syndrome predicts FTLD-TDP, whereas an evolving progressive supranuclear palsy syndrome predicts FTLD-tau pathology. Nonetheless, the same molecular pathology can cause multiple clinical phenotypes. In this brief report, we describe a number of key clinical and imaging features that are correlated with a specific pathological subtype of FTLD-FUS: atypical FTLD-U (aFTLD-U). This entity is thought to account for the majority of tau and TDP-43 negative FTLD cases. ${ }^{3}$

This 44-year-old patient presented with two years of progressive decline in behaviour and cognition. He had no past psychiatric history and, most importantly, no family history of dementia. Personality changes were highly obsessional and ritualistic in nature. An asymmetric $(\mathrm{R}>\mathrm{L})$ pattern of frontotemporal and caudate atrophy was found on brain imaging and post-mortem examination. The progression of his disease
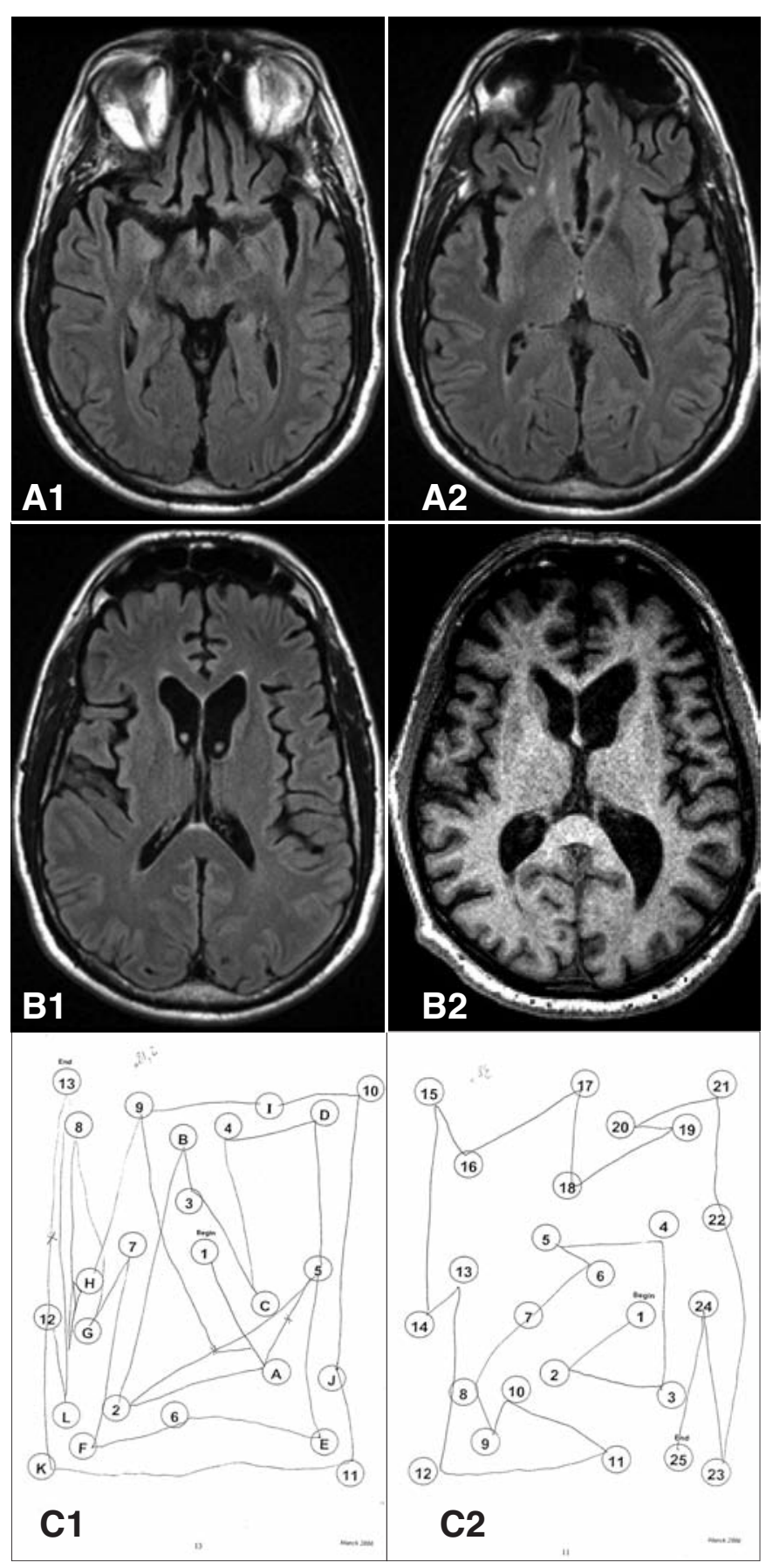

Figure 1: A) Axial T2-weighted FLAIR MRI conducted ten months after his first symptoms reveals asymmetric $(R>L)$ frontotemporal atrophy, particularly of the orbito-frontal, anterior insular, anterior cingulate, and anterior temporal cortices. There was no parietal lobe atrophy, structural lesion, or white matter anomaly. B) The axial sequence on the left is from our patient, and shows asymmetric caudate atrophy, $R>L$. In comparison with a case of FTLD-TDP (right), our patient's caudate nuclei are considerably smaller. This difference is highly significant considering that the patient with FTLD-TDP is 20 years older and was scanned three years after symptoms onset. C) Impaired executive function on Trail Making-B (left) requiring alternation between letters and numbers (i.e. slowed performance, four errors). 


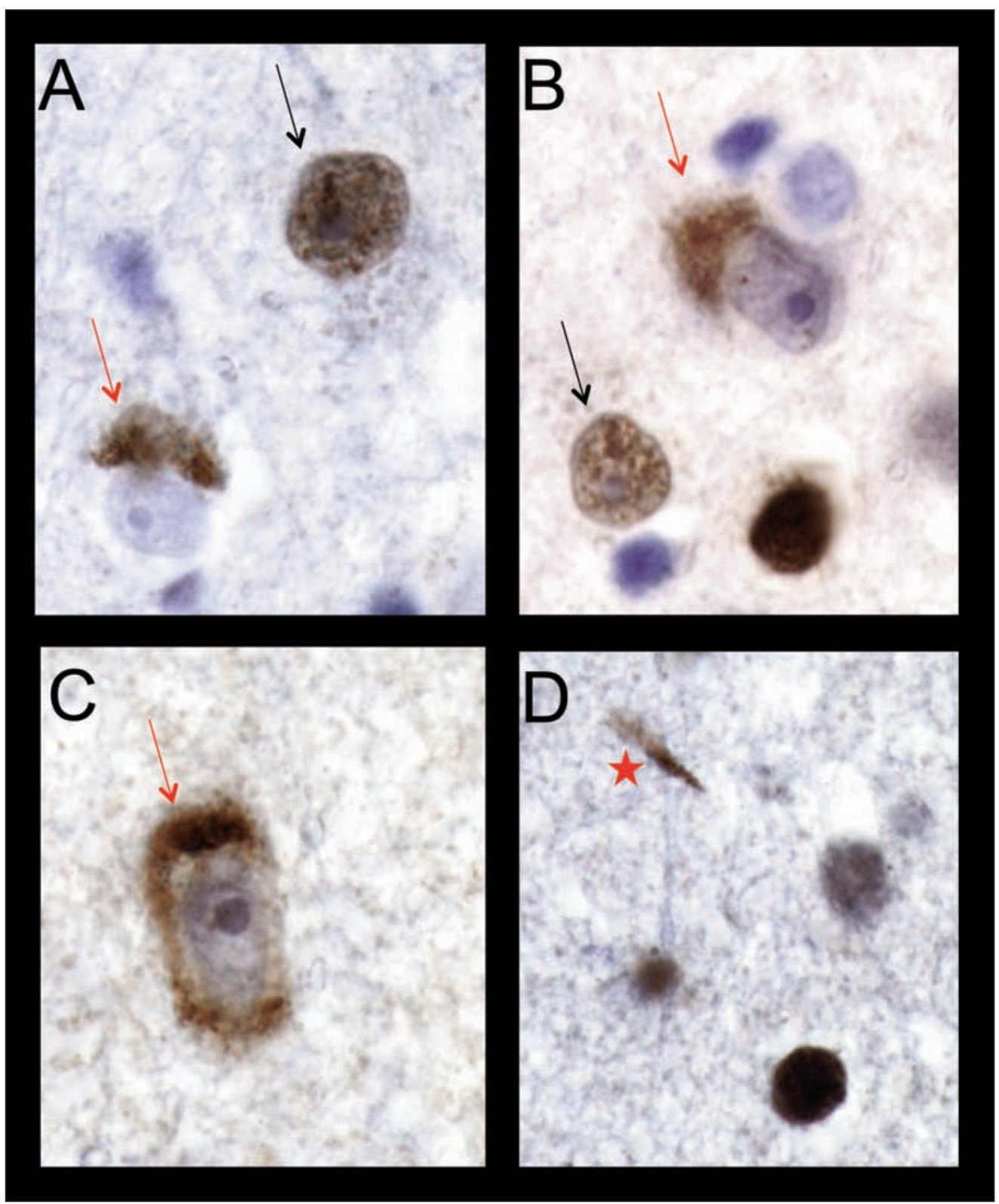

Figure 2: A-C. Neuronal cytoplasmic inclusions that stained positively for FUS protein (red arrows). Unaffected neurons show FUS immunoreactivity restricted to the nucleus (black arrows). D. A short thick FUSpositive dystrophic neurite.

was rapid and not associated with other neurological comorbidities. A psychiatric condition was ruled out by the relentless progression to death. Early age-of-onset Alzheimer's disease is associated with significant memory loss and a posterior cortical pattern of atrophy, both absent here. Most patients with sporadic bvFTD due to FTLD-tau or FTLD-TDP present in the sixth to seventh decade, and many show accompanying oculomotor impairment (tau) or MND (TDP-43). Instead, a number of unusual features suggested that a distinct form of pathology was driving his behavioural changes. This is thought to be the signature of bvFTD secondary to FTLD-FUS (aFTLD-U subtype) pathology.
The identification of FUS-immunoreactive neuronal cytoplasmic inclusions followed the discovery that FUS gene mutations were a significant cause of familial ALS. ${ }^{2}$ FTLD-FUS remains a rare cause of bvFTD, accounting for $5-10 \%$ of patients, with aFTLD-U being the most common subtype..$^{3-4} \mathrm{~A}$ growing number of studies suggests that certain clinical features help discriminate this pathology from other FTLD patients including 1) early age-of-onset bvFTD (mean age of 41 yearsold), 2) absent family history of dementia, 3) obsessional, ritualistic, and stereotyped behaviour-predominant clinical subtype of bvFTD, with one-third showing hallucinations and/or delusions, 4) none or few motor symptoms (3\%), 5) 
frontotemporal atrophy and moderate to severe caudate atrophy on MRI (see Figure 1B), and 6) short disease duration (mean of eight years)..$^{2-4}$ Our patient exhibited each of these six clinical features in addition to intraneuronal FUS-positive inclusions on pathological examination. Further research is needed, however, to determine the specificity and sensitivity of these features.

Behavioural interventions adapted to each individual patient can help in bvFTD, while selective serotonin reuptake inhibitors can improve some compulsive behaviours. ${ }^{5}$ Cholinesterase inhibitors may exacerbate behavioural abnormalities and are not recommended in FTD. Some patients benefit from memantine, and two prospective, placebo-controlled clinical trials are ongoing to evaluate its efficacy. In all patients, physical exercise and a healthy diet are recommended. Our patient's profile is in accord with an increasing body of literature suggesting a distinct phenotype associated with the aFTLD-U subtype of FTLD-FUS. This is very encouraging given its high likelihood for early diagnosis and accurate prediction of underlying molecular pathology during life if recognized in time. Newly developed animal models of FUS pathology are expected to help develop future treatment strategies. Other approaches such as deep brain stimulation should be studied in light of the fact that these patients die at a very young age as a result of their disinhibited behaviours and inappropriate eating habits.

\section{ACKNOWLEDGEMENTS}

The authors thank the patient and his family. We are also grateful to the staff at the Neuropathology Department at UCSF for their help with this case.

This work was funded in part by a Fellowship grant by the FRSQ (Fonds de Recherche en Santé du Québec) to Dr. Robert Laforce Jr.

\section{REFERENCES}

1. Rascovsky K, Hodges JR, Knopman D, et al. Sensitivity of revised diagnostic criteria for the behavioural variant of frontotemporal dementia. Brain. 2011;134:2456-77.

2. Neumann M, Rademakers R, Roeber S, Baker M, Kretzschmar HA, Mackenzie IR. A new subtype of frontotemporal lobar degeneration with FUS pathology. Brain. 2009;132:2922-31.

3. Snowden JS, Hu Q, Rollinson S, et al. The most common type of FTLD-FUS (aFTLD-U) is associated with a distinct clinical form of frontotemporal dementia but is not related to mutations in the FUS gene. Acta Neuropathol. 2011;122:99-110.

4. Urwin H, Josephs KA, Rohrer JD, et al. FUS pathology defines the majority of tau- and TDP-43-negative frontotemporal lobar degeneration. Acta Neuropathol. 2010;120:33-41.

5. Rabinovici GD, Miller BL. Frontotemporal lobar degeneration: epidemiology, pathophysiology, diagnosis and management. CNS Drugs. 2010;24:375-98. 